\title{
Gewalt gegen Rettungsdienstpersonal
}

Robert Ziegenfelder

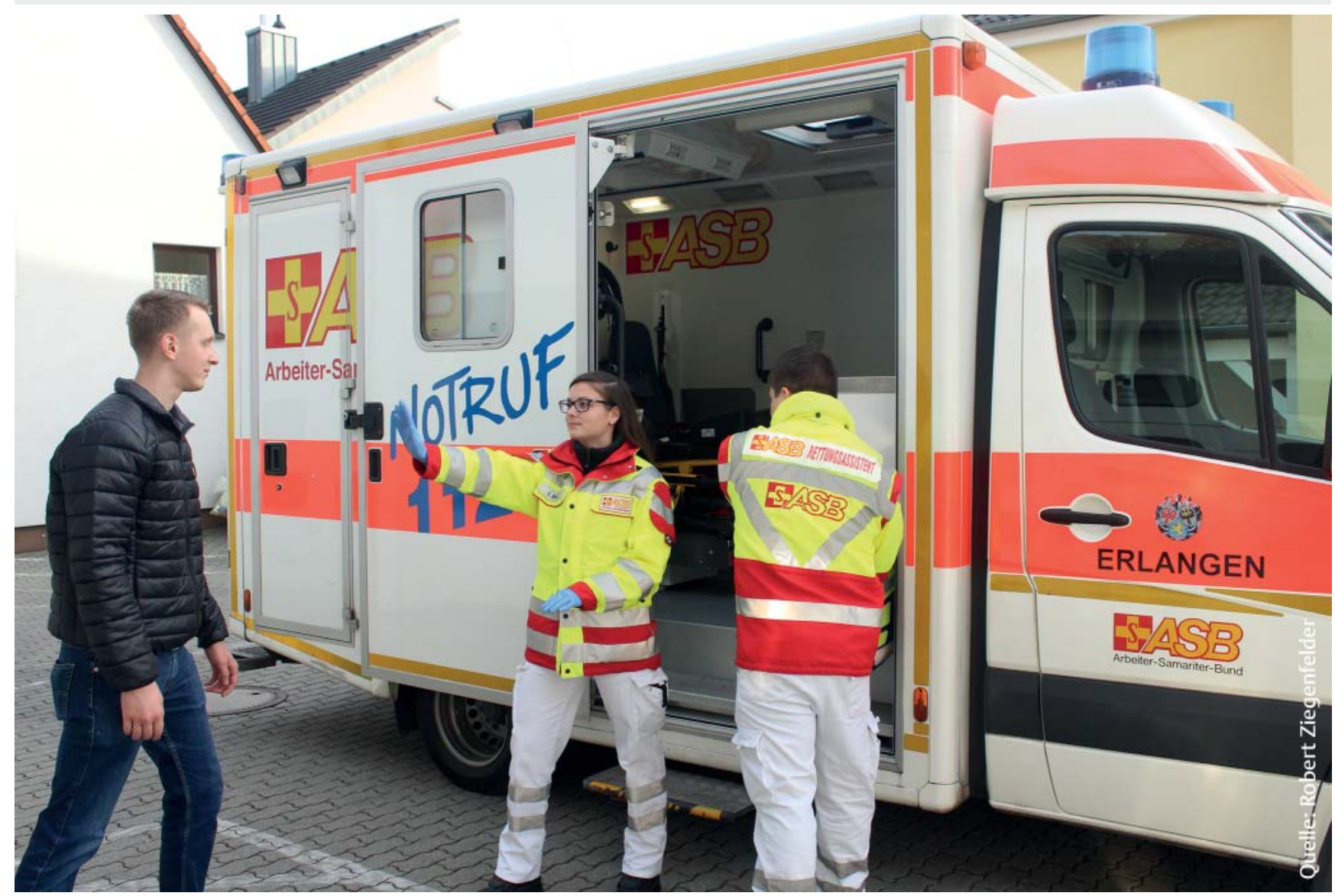

Immer wieder ist Rettungsdienstpersonal mit Konflikten und angespannten Situationen konfrontiert. Dann gilt es eine Eskalation zu verhindern. Nur wie? Dieser Beitrag kann das Thema zwar nicht vollständig aufarbeiten; er schlägt Ihnen aber Lösungsansätze vor, wie Sie in solchen Situationen präventiv agieren können, und macht klar, welche Rolle Ihre persönliche Einstellung spielt.

\section{Umgang mit spannungsgeladenen Situationen}

Familienstreit, psychische Dekompensation, Suizidandrohung, aggressive Grundstimmung gegenüber Einsatzkräften - die Szenarien, in denen es zu Gewalt gegen Rettungsdienstpersonal kommen kann, sind vielfältig.

Die Medien berichten häufig von Übergriffen gegenüber Rettungsdienstpersonal. Sehr viel häufiger ist das Rettungsdienstpersonal mit Situationen konfrontiert, die Strategien verlangen, um eine Eskalation frühzeitig zu verhindern und die Spannung aus der Situation zu nehmen. Derartige Einsätze kommen sowohl im städtischen als auch im ländlichen Einsatzgebiet vor.
Mit welcher Strategie begegnet man solchen Situationen und Szenarien? Wie erkennt man eine sich aufbauende aggressive Haltung gegenüber dem Rettungsdienstpersonal und kann diese in eine angemessene Einsatzatmosphäre wandeln?

\section{Verbale Angriffe gegenüber medizinischem Personal}

Laut Studien wurden 72 - 78 \% der Mitarbeiter in Gesundheitseinrichtungen bereits einmal verbal angegriffen, 28 \% erleben in Deutschland mindestens einmal im Monat massive Gewalt durch Patienten oder Angehörige [1]. Dabei ist es wichtig, dass nicht nur körperliche Ausei- 
nandersetzungen als Angriff zu werten sind, sondern bereits verbale Angriffe belastend sein können.

Einer Anfang 2016 vom Bayerischen Roten Kreuz (BRK) veröffentlichen Studie zufolge wurden im Jahr 2015 Rettungskräfte in 180 Fällen verbal oder körperlich angegangen. Bei 1,7 Mio. Rettungsdiensteinsätzen machen solche Einsatzsituationen also lediglich $0,01 \%$ aus.

Merke

Nur bei sehr wenigen Einsätzen kommt es zu Gewalt gegenüber Rettungsdienstpersonal.

Diese Zahl scheint (bezogen auf die Menge der Einsätze) insgesamt zwar eher gering, dennoch ist eine Steigerung der Übergriffe gegen Rettungsdienstpersonal in Studien nachweisbar. Das bestätigt die Bedeutung präventiver Strategien und die zunehmende Bedeutung deeskalativer Ansätze im Rettungsdienstalltag.

\section{Aggression und Rettungsdienst}

Menschen reagieren auf manche Ereignisse, Erlebnisse und Situationen mit Aggression. In jedem menschlichen System (Partnerschaft, Familie, Verein, Dorf, Arbeitsplatz etc.) kann es zu aggressiven Verhaltensweisen kommen, insbesondere wenn Störungen in diesen Systemen auftreten.

Bei der Arbeit mit Patienten ist das Potenzial für angespannte Situationen aus verschiedenen Gründen deutlich erhöht. Wer weiß, wie Gewalt und Aggression entstehen und wie ihnen am besten begegnet werden kann, ist besser für solche Situationen gerüstet.

\footnotetext{
GRÜNDE FÜR GEWALT UND AGGRESSION IM GESUNDHEITSWESEN

- inneres Spannungsfeld des Patienten

- hohes Aggressionspotenzial des Patienten

- mangelnde Einsicht in Maßnahmen

- Betreuung und Behandlung werden als Gewalt empfunden

- starke und zahlreiche Stressoren für Mitarbeiter

- schwierige Helferbeziehung

- eingeschränkte Kommunikationsmöglichkeit des Patienten

- Erwartungsdruck an Patienten und Mitarbeiter
}

Der Einsatz eines Rettungsteams ist für die betroffenen Patienten fast immer eine Ausnahmesituation. Professionell agierende Rettungsdienstkräfte berücksichtigen die besondere Situation, in der sich ein Notfallpatient und auch seine Angehörigen befinden. Im Verlauf jeder Krankheit, Pflege oder Betreuung gibt es Phasen, in denen Aggression entstehen kann. Ängste, Autonomie- und Selbstwertverluste, Existenzsorgen, persönliche Krisen, Schmerzen, Wut (auf sich selbst oder auf andere) u. a. belasten den Patienten. Er ist verletzlich, hilflos und empfindlich. Diese Kombination erzeugt ein Spannungsfeld, in dem der Betroffene schon auf banale Kleinigkeiten aggressiv reagieren kann - ohne dass dies vom Gegenüber vermutet wird.

Menschen, die sich in einer Krisensituation aggressiv verhalten, sind in der Regel nicht in der Lage, ihre Probleme auf andere Weise zu lösen. Ihre Aggression ist eine ebenso physiologische Reaktion wie z. B. die Flucht in Gefahrensituationen. Deeskalation sollte deshalb immer darauf zielen, (weitere) Provokationen zu vermeiden ( $\bullet$ Abb. 1).

Merke

Aggressionen sind oft der besonderen Situation geschuldet und basieren nicht auf Ablehnung.

\section{Deeskalation als Strategie zur Bewältigung}

Deeskalation bedeutet, Konflikte und sich aufschaukelnde Aggressionen zu verhindern. Sie ist der schwierigste Aspekt des Konfliktmanagements.

Für die Deeskalation gibt es keine Universallösung. Eine Reaktion, die in einer Situation zur Entspannung führte, kann in einer anderen Situation das Gegenteil bewirken. Deshalb gibt es beim Thema Deeskalation eigentlich kein Richtig und Falsch. Der Ansatz geht vielmehr davon aus, dass bestimmte Reaktionen die Wahrscheinlichkeit einer Eskalation senken. So kann schon eine Änderung des Settings die Situation beruhigen ( $\bullet$ Abb. 2).

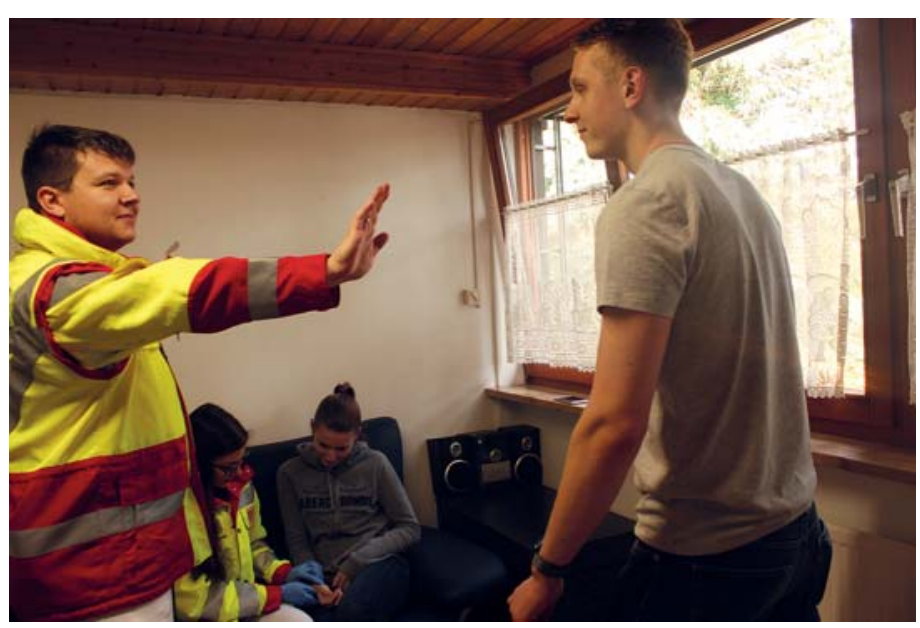

- Abb. 1 Bei der Untersuchung von Patienten sichert ein Kollege den Untersuchenden ab und versucht, den Abstand zu wahren. So vermeidet er Provokationen. 


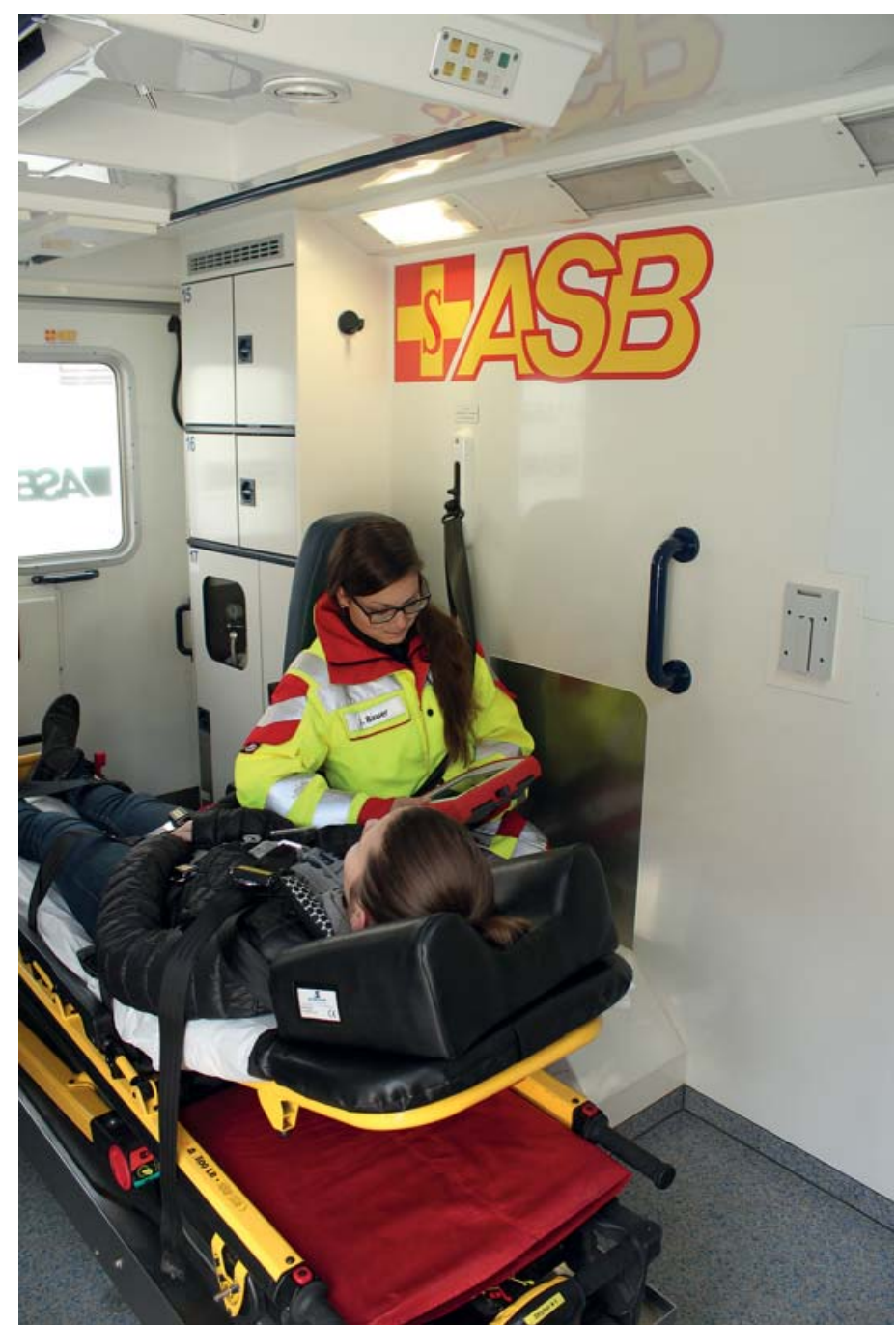

- Abb. 2 Manchmal kann die Änderung des Settings, also die Veränderung der räumlichen Gegebenheiten und der Zahl der Beteiligten, die Situation beruhigen und deeskalieren. In diesem Beispiel sind im Verlauf nur noch die Behandelnde und die Patientin im RTW.

\section{Eskalation durch vermeintliche Hilfsmittel}

Weit verbreitet ist die Annahme, dass persönliche Schutzausrüstung wie eine Schutzweste, Pfefferspray, stichsichere Handschuhe und andere Hilfsmittel die Rettungskräfte schützt und damit Sicherheit bietet. Das Gegenteil ist der Fall. Das offensichtliche Tragen derartiger Gegenstände kann von anderen als Bedrohung verstanden werden und befremdlich wirken. Dies kann eine Eskalation in einer anfangs normalen Einsatzsituation fördern.

Merke

Sichtbare getragene Hilfsmittel wie Westen und Pfefferspray wirken eher eskalierend.

Zweifellos kann in besonderen Einsatzsituationen das Mitführen solcher Gegenstände eine Option sein. Besser ist es jedoch, sich von einer erkennbar gefährlichen Situation fernzuhalten und gerade in brenzligen Situationen mehr Wert auf Eigensicherung und Prävention zu legen.

\section{PRAXISTIPP}

Begeben Sie sich bei entsprechenden Meldungen durch die alarmierende Leitstelle gar nicht erst in den Gefahrenbereich, sondern warten Sie auf eine Klärung durch die Sicherheitskräfte.

\section{Ihre Einstellung zählt}

Rettungsdienstpersonal richtet den Blick während eines Einsatzes oft auf das medizinisch Relevante. Für den Patienten und seine Angehörigen bedeutet ein solcher Einsatz aber noch viel mehr - und das sollte jede Rettungskraft berücksichtigen. Reflektieren Sie auch Ihr eigenes Verhalten - es lohnt sich!

Bedenken Sie: Der Hilfesuchende befindet sich in einer Krisensituation und weiß sich vielleicht nicht zu helfen. Er ist möglicherweise mit der Situation überfordert und hat keine Bewältigungsstrategie. Hinzu kommt, dass viele Einsätze des Rettungsdienstes im häuslichen Umfeld und somit in der Privatsphäre stattfinden. Allein dies kann zu Spannungen führen. Daher kommt es auf die zwischenmenschliche Kompetenz und das Auftreten des Personals an. Seien Sie höflich - auch bei Einsätzen, in denen eine Eskalation wahrscheinlich ist! Das ist der erste Schritt der Deeskalation und sollte die Grundeinstellung im rettungsdienstlichen Einsatz widerspiegeln.

\section{PRAXISTIPP}

Stellen Sie sich dem Patienten freundlich vor und fragen Sie ihn, ob Sie in die Wohnung eintreten dürfen. Das kann Barrieren reduzieren und die Stimmung entspannen.

\section{Verständnis für die Ressourcen des Gegenüber}

Die wichtigste Regel ist also ein höflicher und wertschätzender Umgang - auch in aufgeladenen Situationen. Dieser sollte ergänzt werden durch die Auffassung, dass jeder Mensch in jeder Situation das Bestmögliche aus seinen Ressourcen macht. Der Mensch ist nicht sein Verhalten. Diese Grundannahmen stammen aus dem Kommunikationsmodell NLP (Neuro-Linguistisches Programmieren). Sie haben sich als wertvoll für die Praxis erwiesen und sollten in jeder konfliktträchtigen Situation berücksichtigt werden.

\section{Merke}

Die beste Information über eine Person ist ihr Verhalten. 


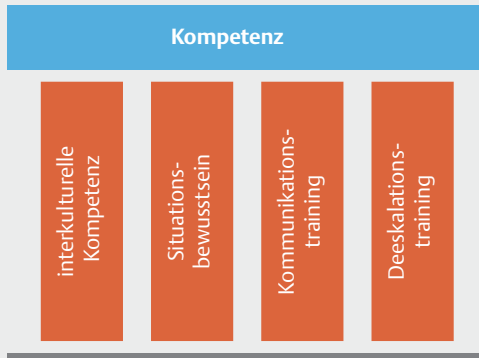

Eigensicherung im Rettungsdienst

- Abb.3 Deeskalation und Prävention durch mehrstufige Schulung.

\section{Weiterbildungen und Schulungen}

\section{Mehrstufiges Ausbildungsmodell}

Deeskalierend aufzutreten kann man lernen. Für die gezielte Schulung der Einsatzkräfte reicht eine einzelne Fortbildungsveranstaltung allerdings nicht aus; sie kann lediglich ein erster Schritt sein. Vielmehr ist eine Weiterbildung und Ausbildung in mehreren Schritten ratsam, die sich am besten auf 4 Säulen stützt ( $\triangleright$ Abb. 3 ). Deeskalation betrifft viele Aspekte, und es braucht Training und Wiederholung, um sie in den entsprechenden Situationen anzuwenden.

Grundsätzlich sind folgende Fortbildungen für Rettungsdienstpersonal empfehlenswert:

- Eigensicherung für Rettungskräfte

- interkulturelle Kompetenz

- Deeskalationstraining

- Kommunikationstraining

Basis sollte eine Schulung zu Eigensicherung und Eigenschutz sein (vgl. retten! 5/2012). Trotz aller präventiven Maßnahmen wird es immer Einsätze geben, die Schutzmaßnahmen erfordern, egal wie gut das Rettungsdienstpersonal deeskaliert.

Aufbauend auf dieser Basis empfiehlt sich die Stärkung der interkulturellen Kompetenz, um sich für den Umgang mit Patienten und Angehörigen fremder Kulturen zu sensibilisieren, Verständnis für andere Denkweisen zu haben, Missverständnisse zu verhindern und so Aggressionspotenzial abzubauen.

Eine dritte Säule ist ein gezieltes Deeskalationstraining. Dort lernen Sie, all das bisher Gelernte anzuwenden und in realistischen Simulationen einzuüben, um in aggressiven Situationen sicher zu handeln. Sie lernen, die eigene Außenwirkung auf andere zu reflektieren, und trainieren Gestik, Mimik, Bewegungen und Eigenschutzmaßnahmen. 
Der vierte, entscheidende Baustein, um die Deeskalationskompetenz zu stärken, ist ein Kommunikationstraining.

\section{Kommunikationsschulung}

Im Kommunikationstraining wird die verbale und nonverbale deeskalierende Gesprächsführung vermittelt. Eine Grundannahme des NLP ist: „Die Bedeutung meiner Kommunikation ist die Reaktion, die ich bekomme.“ Achten Sie also auf Ihre Kommunikation, verbal wie nonverbal - dann sind Sie in der Lage, Situationen zu entspannen.

Techniken u. a. des NLP und anderer Konzepte, wie Pacing und Leading, sind exzellente Werkzeuge, um allein durch geeignete Kommunikation aggressionsbeladene Situationen zu meistern. Die Kommunikationsmodelle nach F. Schulz v. Thun und Paul Watzlawick helfen zusätzlich, in konfliktbeladenen Situationen die Gesprächsführung auf der Sachebene zu halten.

Alle Schulungen sollten darauf hinwirken, ein gesteigertes Situationsbewusstsein zu schaffen und in (potenziell) aggressionsbeladenen Situationen kompetent handeln und auftreten zu können.

\section{Ermittlung des Bedarfs}

Blinder Aktionismus und unstrukturierte Versuche, sich in diesem Bereich selbst fortzubilden, helfen nicht, die eigene Kompetenz zu stärken. Um sich gezielt weiterzubilden, sollten Sie zunächst herausfinden, zu welchen Themen Weiterbildungsbedarf besteht. Erheben Sie dazu die stattgefundenen aggressionsbeladenen Einsatzsituationen sowie die Art der erlebten Gewalt.

Der ASB-Landesverband Hessen hat zusammen mit seiner Mitarbeitervertretung einen Fragebogen zu diesem Thema erstellt und stellt diesen für Rettungsdienstpersonal zur Verfügung (http://www.asb-hessen.de). Damit lässt sich auch einschätzen, bei wie vielen Einsätzen tatsächlich Gewalt auftritt.

\section{Fazit}

Gewalt, verbale und körperliche Übergriffe kommen im Rettungsdienstalltag vor. In Einsatzsituationen mit Gewalt gegenüber dem Rettungsdienstpersonal hilft eine gute Deeskalationskompetenz mehr als ein Selbstverteidigungstraining. Das notwendige Handwerkszeug können Sie in Weiterbildungen lernen, um für diese Situationen sensibel zu werden und das präventive Vorgehen zu stärken.

\section{KERNAUSSAGEN}

- Aggressives Verhalten kommt überall im menschlichen Leben vor - nicht nur im Rettungsdienst.

- Eine vermeintliche Schutzausrüstung wirkt eher eskalierend als deeskalierend.

- Eine positive Einstellung gegenüber dem Patienten und den Angehörigen ist eine Grundvoraussetzung für eine gute Versorgung.

- Deeskalation können Sie in Schulungen lernen. Einzelne Schulungen bringen allerdings keinen langfristigen Erfolg.

- Optimieren Sie Ihre eigene Kommunikation immer weiter.

\section{Interessenkonflikt}

Der Autor gibt an, dass kein Interessenkonflikt besteht.

Über die Autoren

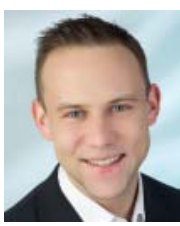

\section{Robert Ziegenfelder, MSc}

ist Leiter Rettungswache bei der ASB Notfallhilfe gGmbH Erlangen und Notfallsanitäter. Er ist NLP-Trainer und Kommunikationstrainer für das österreichische Institut c10plus.

Korrespondenzadresse

Robert Ziegenfelder

Schillerstraße 17

91074 Herzogenaurauch

R.Ziegenfelder@asb-notfallhilfe.de

Literatur

[1] Bärsch T, Rohde M. Deeskalation in der Pflege. Gewaltprävention, Deeskalierende Kommunikation, SaFE- und Schutztechniken. 2. Aufl Books on Demand; 2013

[2] Mittler D. Rotes-Kreuz-Studie: Gewalt gegen Sanitäter nimmt zu. Süddeutsche Zeitung 27.1.2016

[3] Wesuls R, Heinzmann T, Brinker L. Professionelles Deeskalationsmanagement (ProDeMa) - Praxisleitfaden zum Umgang mit Gewalt und Aggression in den Gesundheitsberufen. 4. Aufl Stuttgart: Unfallkasse Baden-Württemberg; 2005

\section{Bibliografie}

DOI http://dx.doi.org/10.1055/s-0042-114130 | retten 2017; 6: 12-16 (c) Georg Thieme Verlag KG Stuttgart · New York ISSN 2193-2387 